\title{
Los planteamientos de Ellacuría sobre la dialéctica de verdad y justicia
}

\author{
Hugh LACEY \\ SWARTHMORE. COLLEgE \\ Traducción: Luis Alvarenga
}

Cuando escribía durante la guerra, Ignacio Ellacuría se preguntaba: “¿Es posible una universidad distinta?". Intentaba demostrarlo volviendo a moldear la universidad de la cual era Rector. Buscaba una universidad que respondiera de manera imaginativa y contundente a la pregunta: ¿Cómo puede integrar la universidad sus tareas medulares y los valores que la fundamentan, con la necesidad de hacer una contribución, del tipo que fuera, para lograr mayor justicia en un contexto donde la mayoría de la gente está sufriendo profundamente, dada la falta de respeto a los derechos humanos? La pregunta se presta a generalizaciones que tienen relevancia en cualquier lugar. Fundamentalmente, involucra a la dialéctica de verdad y justicia. El tomarla en serio y ocuparse de ella de una forma rigurosa tanto en los planes de estudio, como en la investigación y en las actividades de enseñanza, así como en los programas de proyección social, constituye el corazón de la contribución específica que la universidad puede hacer a la búsqueda de la paz. Una universidad fiel a sus valores medulares hará esta contribución. A pesar de escribir en medio del contexto de la guerra, Ellacuría no estaba tan absorbido por sus exigencias inmediatas como

Los planteamientos de Ellacuría sobre la dialéctica de verdad y justicia 
para dejar de lado problemas de urgencia universal. Al contrario, estos últimos resaltaron ante sus ojos la importancia de comprometerse en la formulación de un pensamiento profundo y original.

\section{Los principales planteamientos de Ellacuría sobre la universidad}

No intentaré hacer una exposición sistemática sobre el pensamiento de Ellacuría sobre la universidad y las raíces de éste en la teología de la liberación². En vez de ello, ofrezco una selección de variaciones sobre sus principales temas, los cuales, según mi interpretación, se vinculan con las siguientes ideas:

(1). Los valores centrales de la universidad son (en interacción dialéctica) la búsqueda de la verdad y la formación de los estudiantes para lograr vidas productivas y plenas al servicio del bien común.

(2). La universidad, en tanto universidad, no debe subordinar lo anterior a valores políticos, religiosos, económicos o de otro tipo. Sin embargo, la universidad, en cuanto institución dentro de la sociedad, depende de condiciones materiales, financieras y sociales, cuya disponibilidad puede necesitar de la cooperación universitaria con algunos proyectos que se encuentren en tensión con estos valores.

(3). El objetivo central de la investigación es la realidad concreta -la "realidad histórica": esbozar sus características actuales y variaciones, analizar sus causas, tratar de identificar el rango de posibilidades que lo actualmente realizado otorga para el futuro. La realidad histórica incluye los fenómenos significativos socialmente del tiempo de cada uno, esos fenómenos de los cuales ninguna vida se encuentra desligado, y las respuestas a los mismos, las cuales definen el carácter moral del tiempo de cada individuo.

(4). La verdad no puede buscarse al margen de las vidas y las relaciones sociales de los investigadores, maestros, estudiantes, etc. Debe buscarse en relación dialéctica con otros valores: cualquiera de los que están incorporados en grado sumo dentro de la sociedad (verbigracia, los del mercado, la propiedad privada y el consumismo), o aquellos de (por ejemplo) la 
justicia, la búsqueda de la paz y la solidaridad con los pobres ${ }^{3}$ (los cuales son, según la perspectiva teológica de Ellacuría, los valores del Reino de Dios).

(5). Algunos principios fundamentales:

(a). Para entender lo real, uno debe buscar qué es lo posible, dados los condicionamientos de lo actual.

(b). Hay una dialéctica compleja entre lo personal y lo social.

(c). Las posibilidades de lo nuevo (y liberador) podrían estar presentes entre los pobres.

(d). La perspectiva de los pobres es esencial para adquirir una comprensión rigurosa de la realidad.

\section{Posibilidades futuras}

Lo que encuentro más llamativo en este punto es el énfasis que hace Ellacuría en las posibilidades futuras. Esto no refleja únicamente su juicio sobre la falta de adecuación moral de lo que se está realizando en las sociedades actuales, por ejemplo, el grosero irrespeto a los derechos humanos que él encontraba diariamente en El Salvador, sino también su aguda conciencia de la necesidad moral de identificar posibilidades de liberación y de movimientos que pudiesen actualizar dichas posibilidades. Moverse hacia la actualización de mayores posibilidades liberadoras es un problema que demanda la investigación académica, así como ganar conocimientos (iAquella lucha ajena al conocimiento relevante no puede aspirar a producir resultados liberadores!). La investigación académica es asunto de la universidad. Ellacuría quería colocar en el centro del plan de estudios y de los esfuerzos de investigación universitarios la siguiente pregunta: ¿Cuáles son las posibilidades liberadoras a las que se puede aspirar razonablemente y cuáles son los medios que pueden conducir hacia su actualización? Y quería que esta pregunta fuera abordada con las más rigurosas herramientas de investigación. Inclusive, en medio de la guerra, Ellacuría no subordinó esta tarea a las exigencias de la lucha inmediata o al compromiso con alguna de las partes beligerantes. Más aún, para él esta tarea implicaba abordar posibilidades remotas: un mundo donde los derechos humanos sean completamente respetados y un mundo

\section{9}

Los planteamientos de Ellocuria sobre la dialéctica de verdad y justicio 
de paz, para el cual él anticipó el horizonte utópico de una "civilización del trabajo", que podría reemplazar a la actual "civilización del capital ${ }^{+}$.

\section{Epistemología}

El principio formulado en los siguientes términos "para comprender lo real, uno debe buscar qué es lo posible, dados los condicionamientos de lo actual", es un importante principio epistemológico. (Esto se halla enlazado inextricablemente con la descripción y la explicación de las realizaciones actuales - la comprensión de un fenómeno implica, interactivamente, describir, explicar y sintetizar las posibilidades que el fenómeno permite ${ }^{5}$. Conocer es aprehender lo real, pero lo real no es idéntico a lo actual, puesto que en el futuro se pueden actualizar las posibilidades que ahora están sin actualizarse. Lo real abarca tanto lo posible como lo actual. Las posibilidades novedosas pueden estar presentes en una forma anticipatoria dentro de lo actual, y las posibilidades novedosas pueden incluir posibilidades liberadoras, mejores integraciones de la justicia, trayectorias hacia la paz. Con este principio, Ellacuría evitó tanto el determinismo - que afirma que lo posible se encuentra ínsito en lo actual-, como el voluntarismo - el cual sostiene que lo posible no está condicionado por lo actual-. Lo actual condiciona y a la vez posibilita la actualización de novedosas posibilidades futuras.

\section{Metodología}

Dado este principio epistemológico, surge una pregunta metodológica: ¿Cómo podemos investigar -empírica, científicamente, con rigor intelectual- las posibilidades reales que no han sido actualizadas? Esta pregunta se encuentra reforzada por el hecho de que los datos empíricos (informes de las observaciones) provienen necesariamente de lo que está actualizado. En consecuencia, no se toma en serio este principio si se limita la investigación empírica a las estructuras dominantes de la actualidad, así como sus regularidades y sus tendencias, y a partir de esto se asume que esas tendencias necesariamente continuarán en el futuro. Hacer eso excluiría, metodológicamente hablando, la identificación de las posibilidades novedosas. También sería una receta para la desesperación entre quienes padecen la injusti- 
cia. Nótese que en las ciencias naturales podemos lograr una capacidad de penetración en las posibilidades que no están actualizadas hasta el momento por medio de la metodología experimental. No podemos hacer lo mismo, empero, cuando queremos poner a prueba las posibilidades de las estructuras sociales puesto que son sistemas abiertos e interactivos que forman parte de una red de relaciones mundiales.

Una metodología que pueda incorporar adecuadamente el principio epistemológico anterior necesita ser consistente con los siguientes supuestos: (a) Las regularidades descubiertas en la conducta humana de la mayoría no representan leyes generales. Más bien, son tan sólo regularidades conductuales verificadas actualmente puedan cambiar en función de las transformaciones sociales ${ }^{6}$. (b) Siempre ocurre que algunas de las conductas de ciertas personas no pueden subsumirse bajo las regularidades dominantes en la actualidad. Estas regularidades admiten anomalias, de tal suerte que el poder insticucionalizado nunca es capaz de suprimir todas las fuentes alternativas de cambio social. Estas anomalías actuales pueden constituirse en fuente de posibilidades sociales que podrían desarrollarse considerablemente en el futuro. Nada de esto contradice lo anteriormente señalado, en el sentido de que las estructuras dominantes condicionan de modo significativo el tipo de posibilidades futuras, pero que no por ello las pueden abarcar en su totalidad'.

Dados estos supuestos, debemos poner atención a las anomalias a fin de hacernos con una aprehensión adecuada de las posibilidades futuras [Principio 5, literal (c)]. Por consiguiente, se necesita la investigación empírica para identificar aquellos grupos cuyas prácticas, movimientos y comunidades representan, por anticipado, las posibilidades liberadoras y la incorporación de valores "alternativos", con capacidad probada de llevar a una incorporación aún más plena. Ignacio Martín-Baró, colega de Ellacuría, señala los valores de "la solidaridad y cooperación, sobriedad y persistencia, sensibilidad y capacidad de sacrificio" que encontró entre los miembros de las "organizaciones populares", en tanto ejemplos de dichos valores alternativos". Ellacuría también identificó a las organizaciones populares de América Latina, así como a las Comunidades Eclesiales de Base, como grupos anómalos clave que anticipan posibilidades liberadoras.

Podría objetarse: Fllacuría está equivocado, por cuanto en el presente no hay posibilidades genuinas (a las cuales valdría la pena aspi-

Los planteamientos de Ellocuria sobre la dialéctica de verdad y justicia 
rar) fuera de las estructuras del mercado, la propiedad privada, la democracia formal y la sombra del poder militar estadounidense. iQuizá! Pero uno no puede afirmar esto sin investigar empíricamente las dinámicas de los grupos anómalos ${ }^{9}$. Sin embargo, podría haber razones para pensar que se equivocó: la inercia de las instituciones y la apertura de las instituciones actualmente dominantes para dar cabida a reformas limitadas, la utilización del poder para defender los intereses dominantes, la difundida internalización de los valores individualistas en la actualidad y la creencia en que éstos surgen de la "naturaleza humana”. El contexto de su vida y muerte da fe de que Ellacuría estaba muy familiarizado con estos argumentos - pero también con las evidencias que los ponen a prueba-.

No podemos conocer el futuro con certidumbre. Cualquier proyección que hagamos sobre él tiene un núcleo irreductible de conjeturas y debe tomarse en cuenta que nosotros somos algunos de los agentes que podrían realizarlo. Las proyecciones sobre el futuro son una mezcla entre la promesa y la predicción, entre la anticipación y la intención. Hay una forma común de "realismo" que insiste en que el futuro estará (más bien deberá estar) expresado en las instituciones dominantes de la actualidad. Por supuesto que cuando aquellos que ocupan sitios privilegiados en las instituciones dominantes insisten en este punto, probablemente lo que quieren decir es que están "prometiendo" usar su poder para garantizar esto, o para anticipar que los agentes de los proyectos alternativos no pueden tomarse en cuenta para consumar un orden más justo, o que ellos no arriesgarán sus vidas en compromisos que podrían ser necesarios para alcanzar un futuro distinto.

\section{El significado de las organizaciones populares}

Contra este tipo de "realismo", Ellacuría contrapropone: (1) Estas instituciones acuerpan un ideal moral deficiente. (2) Las instituciones capitalistas no pueden ser universalizadas, tanto por razones sociales como ecológicas. Socialmente, su expansión (verbigracia, con la embestida del actual proyecto neoliberal) implica cometer tremendas violaciones a los derechos humanos, mientras que desde el punto de vista ecológico, el mundo no puede costear, para todos, un estándar de vida comparable al que se posee mayoritariamente en los países tecnológi- 
camente avanzados. (3) Los grupos que efectivamente abracen los valores no individualistas son posibles; lo sabemos porque ya existen, y están encarnando esos valores en cierta medida, tanto en las organizaciones populares como en las comunidades cristianas de base.

En este punto, nos podría ayudar un poco una reflexión. En tiempos recientes, las organizaciones populares han estado discutiendo sus valores, cómo estos se contrastan con aquellos propios de las políticas y programas neoliberales, y los programas en los cuales sus valores puedan implementarse. En el Foro Social Mundial, llevado a cabo en Porto Alegre, Brasil, en febrero de 2002, los valores "alternativos" relevantes se calificaron como "de participación popular". Yo los incluí dentro de la siguiente lista: solidaridad y compasión en vez de individualismo; el bien social como elemento de equilibrio de la propiedad privada y las ganancias; el control de los objetos naturales subordinado a la sustentabilidad ecológica; la no violencia practicada a un punto tal que no se tolere la injusticia; el bienestar de todas las personas por encima de la primacía del mercado y la propiedad; el fortalecimiento de una pluralidad de valores en vez de una co-modificación cada vez más extendida; la liberación humana, abarcando y modulando la libertad individual y la eficiencia económica; una veracidad que aspira lograr un entendimiento comprensivo del sitio que ocupan nuestras vidas en el mundo, que busca identificar las posibilidades liberadoras ocultas dentro del orden predominante y que no identifica lo que es posible con las principales tendencias de dicho orden; disposición para someter a la crítica y la investigación los presupuestos legitimadores de las propias prácticas (compromiso con la transparencia), en vez de colocar estos presupuestos entre las "certezas" que son vistas como más allá de la investigación; los derechos de los pobres y la primacía de la vida priorizados sobre los intereses de los ricos; la dimensión participativa como elemento que abarca la democracia formal; y los derechos civiles y políticos en relación dialéctica con los derechos sociales, económicos y culturales. ${ }^{10}$

La existencia actual de organizaciones populares, que buscan profundizar la encarnación de dichos valores, y el incremento de éstas alrededor del mundo, se constituyen en una opción para otros, incluyendo a la gente que está en la universidad: Solidarizarse con ellas o no; poner o no al servicio de la actualización de las posibilidades que ellas representan la dimensión de ser agente causal que cada uno po-

Los planteamientos de Ellacuría sobre la dialéctica de verdad y justicio 
see. El que estas posibilidades se concreten o no lo hagan depende en un grado significativo de las elecciones que hagan las personas y las instituciones (incluyendo las universidades).

\section{Investigando las posibilidades liberadoras}

En la universidad diferente que Ellacuría buscaba implementar, la búsqueda de la verdad está ligada con la exploración disciplinada de las posibilidades liberadoras y esto presupone aprehender -empírica y teóricamente- las realidades sociales actuales desde la perspectiva de aquellos que necesitan liberarse (Tema 4): ¿De qué posibilidades predominantes se desea una liberación? ¿Qué explica el que estas condiciones se mantengan en las disposiciones sociales actuales? ¿Quiénes son los agentes de estos proyectos - aquellos a los que Jon Sobrino llamó "los pobres con espíritu" y quienes comparten ese espíritu-?" ¿Cuáles son los obstáculos que enfrentan? ¿Pueden superarse esos obstáculos? Buscar institucionalmente la dialéctica de verdad y justicia tiene consecuencias de largo alcance para las actividades docentes e investigativas de la universidad, y éstas consecuencias pueden interpretarse de varias maneras.

\section{Implicaciones para el contenido del curriculum}

$\mathrm{Al}$ reconocer que mi interpretación de ninguna manera excluye otras, sugiero que las implicaciones - basadas en el punto de vista de comprender lo afirmado arriba (Tema 5)'- - puede llevar a colocar cerca de la médula del currículum los siguientes cinco grupos de preguntas, entendidas como problemas para una investigación disciplinada y multidisciplinaria.

(1) ¿En qué maneras concretas se manifiesta el sufrimiento en el mundo actual -y especialmente, dentro de la ubicación propia? iFntre los pobres? ¿Entre los que tienen comodidades materiales? ¿De qué forma se violan los derechos humanos (económicos, sociales y culturales, así como los civiles y políticos)? ¿Cuáles son las diversas dimensiones del sufrimiento? ¿Cómo están conectadas entre sí? ¿Cómo varían en forma e intensidad: como la raza, según género, edad, nivel educativo, situación laboral, nacionalidad, cultura, geografía, entorno, y vitalidad de la comunidad local? 
(2) ¿Cuáles son las distintas (e interactuantes) causas de ese sufrimiento y de las violaciones a los derechos humanos? ¿Hasta qué punto son una herencia del pasado o están derivadas de la naturaleza? ¿De las deficiencias personales, las deformidades personales o los vicios, que podrían ser compartidos por muchos? ¿Del abuso del poder? ¿De los credos religiosos fundamentalistas? ¿Del dominio imperial? ¿De las estructuras socioeconómicas que dominan el mundo actual? ¿Cuáles son los impedimentos para lidiar con esas causas y, por tanto, los obstáculos para lograr una experiencia más plena de bienestar humano? ¿En qué medida las tendencias, las reformas potenciales y las innovaciones que se esperan de la sociedad actual pueden aumentar o remediar los sufrimientos?

(3) ¿Cuáles son las aspiraciones de aquellas personas que sufren, especialmente los pobres y marginados? ¿Cómo la gente pobre se caracteriza a sí misma y que respuestas esbozan para las preguntas anteriores a la luz de su experiencia? ¿Cómo diagnostican las causas de su condición? ¿Cómo expresan sus esperanzas, y cómo identifican y articulan las posibilidades que consideran que están a la altura de sus aspiraciones? ¿Quiénes son sus dirigentes? ¿Quién articula mejor sus aspiraciones de una manera más auténtica y de manera más autorizada? ¿Cuáles son sus propuestas, movimientos y prácticas transformadoras? ¿Qué valores encarnan? ¿Cómo podemos compartir los programas educativos con los pobres, colaborando con sus movimientos y sus deseos, de tal manera que se puedan formular mejor estas preguntas? Así las cosas, ¿es mejor estar conscientes de la realidad concreta de los pobres y estar en disposición de colaborar con ellos, u ofrecerles servicio de manera auténtica? Estas preguntas cobran mayor significado (Principio 5, literal d), si uno acepta que la gente misma, cuando ejercita su poder crítico, es la mejor articuladora de su propia realidad vivida —cuando el ejercicio de su poder crítico la lleva a cobrar una consciencia más clara de las posibilidades a las que está abierta en el futuro, verbigracia: con respeto al crear instituciones que nutren la cooperación y el servicio para otros, o al crear medios de lucha efectivos y no violentos, los cuales son posibilidades que podrían no estar al servicio de los intereses de las élites y que por lo tanto no están consideradas dentro de las corrientes principales del análisis científico social. ¿Dónde están las fuentes de la transformación liberadora - las anomalias - en el entorno propio? (Principio 5, literal c)? 
(4) ¿Depende el estilo de vida del personal universitario y los graduados de las estructuras socioeconómicas que mantienen o aumentan la degradación de los pobres? ¿O de estructuras que generan y mantienen las múltiples patologías sociales que deslucen al mundo (y al país) hoy día, verbigracia: el desamparo, las drogas y la violencia galopante? ${ }^{13}$ ¿Cuáles son las condiciones materiales y sociales de las formas de vida del personal universitario y de los graduados? ¿Requieren que otros sean oprimidos y alienados? ¿Aumentan la destrucción irreversible de la naturaleza? ¿Pueden cambiarse? ¿Cómo? ¿Con qué prácticas? ¿Con qué consecuencias? ¿Se garantizarían esos cambios en vista de los logros positivos que se logran bajo dichas condiciones y los intereses de los que las mantienen?

(5) ¿Cuáles son los valores predominantes que actualmente encarna la sociedad? ¿Los dirigentes políticos los articulan y legitiman? ¿Están esos valores que ellos articulan encarnados en las instituciones sociales? ¿Cómo se comparan con los valores de la "participación popular"? ¿Cuáles son las posibilidades de avanzar ahora - como personas que participan en las instituciones sociales - hacia una encarnación más plena de estos últimos valores? ¿Cuáles son los requisitos psicológicos y espirituales y cuáles los impedimentos para moverse en esa dirección?

Según esta sugerencia, el contenido del currículum universitario debe formularse preguntas como estas en aras de responder a la dialéctica de verdad y justicia, a fin de investigar cuáles pueden ser las posibilidades futuras de justicia, paz, liberación y de una "civilización del trabajo" (Tema 3). Mi sugerencia no está encaminada a proporcionar una alternativa desarrollada para el currículum actual. Todas las preguntas que hemos formulado necesitan fundamentarse por medio del mejor pensamiento proveniente de las disciplinas fundamentales y sus respuestas necesitan comprobarse rigurosamente, confrontándolas con las respuestas obtenidas con distintas metodologías desde las perspectivas morales más contrapuestas; a la vez, buscar estas respuestas añadirá profundidad a las disciplinas y las capacitará para captar los fenómenos morales significativos del propio tiempo. Mi intención es que las preguntas formuladas tengan un alcance tanto nacional como mundial, sin perder su enfoque local, por cuanto lo local es una parte de las redes causales más amplias: no podemos entender lo local sin aprehender su lugar en las estructuras socioeconómicas que tienen pro-

\section{6}

Realidad 90,2002 
porciones globales y no podemos entender ni valorar moralmente las redes mundiales sin estar conscientes de sus efectos en numerosas localidades.

\section{Contacto con las anomalías sociales}

El formularse estas preguntas en una forma empírica disciplinada requiere un contacto genuino con la realidad actual y el sufrimiento de los pobres, así como con los movimientos de cambio que se dan entre estos últimos, un contacto que es inmediato, participante, que expresa la compasión y la solidaridad y que está articulado críticamente. Sin este contacto, no se tiene un lugar adecuado (desde el cual se pueda aprehender la perspectiva de los pobres-Principio 5, literal d) para la investigación empírica que pueda hacer posible el descubrimiento de los efectos negativos de las instituciones dominantes y la identificación de las anomalías, las fuentes de las alternativas sociales prometedoras. El contacto es necesario en aras de la tarea de la universidad de llegar a comprender la realidad social; pero no es suficiente: llegar a esta comprensión es algo que también necesita recursos teóricos y prácticas metodológicas adecuados.

Es indudable que, a fin de contactarse adecuadamente, es necesario desarrollar proyectos de servicio, participación y colaboración en el propio contexto inmediato. Tales proyectos aparecen así como parte integral de la búsqueda de la verdad, y no simplemente como actividades meritorias, suplementarias y opcionales del estudiante, el maestro o el investigador. Son proyectos dentro de los cuales el llegar a comprender las alternativas sociales y los esfuerzos compasivos y solidarios son momentos (tanto cognitiva como moralmente) integrales y mutuamente reforzados de un mismo proceso de liberación (ique es, por tanto, la dialéctica de verdad y justicia!). Son medios para llevar a la universidad a una interacción constructiva con las comunidades en las que hay sufrimientos que imploran por liberarse y donde hay anomalías, fuentes de posibilidades alternativas. En el fondo, esta interacción necesita ser institucionalizada (aunque con apertura), de tal suerte que la universidad no pueda ignorar que la búsqueda de la verdad incluye la tentativa de identificar posibilidades liberadoras (y que nuevos tipos de carreras se puedan abrir para los graduados). 
¿Cómo puede hacerse esto? En otra parte ${ }^{14}$, al reflexionar sobre cómo las actividades de servicio pueden integrarse en el despliegue dialéctico de verdad y justicia, mis colaboradores y yo sugerimos que dichas actividades deben ser ordinariamente parte de un conjunto de programas bien planeados, donde las actividades y los programas abarcan los cuatro siguientes niveles interactivos:

$\$$ Cada uno de los programas y de las actividades tiene un valor por sí mismo en virtud de su esfuerzo por enfocarse en una necesidad que han identificado los miembros de una comunidad de un barrio urbano pobre - llevar recursos, habilidades, capacitaciones, y, por encima de todo, conocimiento y la capacidad de generar conocimiento dentro de la comunidad-.

$\mathbb{S E s t a ́ n ~ i n t e g r a d o s ~ e n ~ u n ~ p r o c e s o ~ d e ~ a m p l i o s ~ c a m b i o s ~ c o m u n i t a r i o s ~}$ (y sociales, si ello fuera posible) - construyendo instituciones que todos los participantes integrarán-dirigido hacia las metas establecidas con los miembros de la comunidad.

$\mathbb{S}$ Se llevan a cabo en lugares donde los estudiantes y otras personas pueden ofrecer servicios comunales que han sido aprobados por la comunidad y que están sujetos a una supervisión y evaluación abiertas; y donde los esfuerzos se realizan a fin de promover la discusión y la interacción entre los miembros de las comunidades y aquellas personas comprometidas en las actividades de servicio, para alimentar el respeto y la amistad y para explorar en conjunto mayores formas de colaboración.

$\$ Están conducidos con un espíritu de reciprocidad, donde todas las personas que participan conciben lo que están haciendo como parte de una tarea común cuyas metas son importantes para todos. El personal universitario, los vecinos de la comunidad y los representantes de otras instituciones, públicas, privadas y comunales, se conciben a sí mismos como parte integrante de un esfuerzo conjunto por los mismos fines mediante papeles diferentes. Los miembros del personal universitario no son asistentes ni proveedores, sino que acompañan y participan en el proceso de cambio social a largo plazo, buscando, entre otras cosas, crear un nuevo tipo de institución educativa, donde la gente pobre pueda participar integralmente y a través de la cual esa 
gente pobre pueda adquirir conocimiento e investigaciones para fundamentar sus proyectos de cambio social. ${ }^{15}$

Ofrezco esta afirmación como una propuesta para la reflexión crítica que debe confrontarse con las prácticas llevadas a cabo en la UCA y otras instituciones que se han inspirado en las ideas de Ellacuría. Nótese que el servicio per se no necesita interactuar positivamente con la búsqueda del entendimiento y, si las condiciones propicias no están dadas, incluso podrían obstaculizarlo, puesto que el contacto rápido y superficial, sin importar cuán bien intencionado sea, puede reforzar fácilmente los estereotipos o llevar a que los pobres se consideren como simples medios para los fines de la universidad.

\section{La interacción del análisis epistemológico y el juicio moral}

La búsqueda de un nuevo tipo de universidad que Ellacuría emprendió se deriva tanto del análisis epistemológico como del juicio moral que los proyectos que buscan ahondar las posibilidades liberadoras en un mundo marcado por graves injusticias son problemas acuciantes de una gran urgencia. El juicio moral permite enfocarse en la búsqueda del entendimiento de la realidad social actual; los resultados de la búsqueda deben dar forma a los proyectos liberadores. Al mismo tiempo (Principio 5, literal b), actualizar a la universidad podría requerir el cultivo de determinadas virtudes: valentía, constancia, franqueza, compromiso, esperanza, humildad, la capacidad de amistarse con la gente y de entender sus diferentes opciones de vida y sus distintas aspiraciones, la capacidad de cultivar el diálogo que, a cambio, puede propiciar la comprensión mutua y el sentido de un futuro compartido en donde todos puedan experimentar un hálito de bienestar, una sensibilidad crítica que es capaz de discernir cuáles movimientos encarnan los valores de la "participación popular" y hasta qué punto lo hacen (partiendo del reconocimiento que no hay un movimiento actual que pueda identificarse plenamente con la aspiración a la liberación), y una fe inquebrantable en la veracidad. Estas virtudes deben construirse sobre una profunda espiritualidad, a la cual deben reforzar ${ }^{16}$. El compromiso es crucial, incluso ante la inseguridad y la incertidumbre, por cuanto las posibilidades liberadoras no pueden actualizarse o, como sucede con frecuencia, anticiparse tan siquiera, sin que para ello concurran las acciones comprometidas de numerosas

Los planteamientos de Ellocuria sobre la dialéctica de verdad y justicia 
personas (aunque ninguna acción comprometida, sin importar su magnitud, tiene garantizado el éxito). Inevitablemente, la probabilidad de que las posibilidades liberadoras sean actualizadas, se ve difusa. Esto provoca que mucha gente se sienta desmotivada. Algunos dicen: "Si tan sólo pensara que hubiera una probabilidad razonable de éxito, yo me comprometería en los movimientos de liberación". Pero una probabilidad razonable de éxito no puede predecirse si se toma como algo anterior o independiente del compromiso. El compromiso es un factor clave que aumenta esa probabilidad, así como moldea el carácter concreto de las posibilidades liberadoras actualizadas.

La idea de que cultivar esas virtudes puede ser necesario para lograr una comprensión adecuada de los fenómenos sociales, se contrasta fuertemente con las nociones comunes que han tomado un lugar preeminente en las reflexiones sobre la metodología de las ciencias naturales. Estas nociones urgen que la búsqueda del entendimiento requiera una postura de distanciamiento, desinterés y un escepticismo previo (junto al compromiso de seguir la evidencia a dondequiera que conduzca y poner a prueba rigurosamente cualquier afirmación que pudiera proponerse) de parte del investigador a fin de evitar la subordinación de las demandas del conocimiento a los valores sociales y morales (o a las perspectivas metafísicas y religiosas).

Ellacuría y yo convenimos que las demandas del conocimiento no deben subordinarse a los valores morales (Tema 2). iLo que es no puede derivarse de lo que es bueno o de lo que debiera ser! En el campo social, sin embargo, la manera de hacer esto no es adoptar una posición de desinterés. El que las posibilidades puedan ser actualizadas depende considerablemente en las opciones humanas y en los valores que estas manifiestan. El que el investigador se abstenga de comprometerse equivale a asumir en los hechos que las instituciones actualmente dominantes continuarán en el futuro; sin un compromiso moral apropiado, uno no puede encontrar las anomalias de tal forma que puedan ser una fuente de datos empíricos. Irónicamente, la investigación "desinteresada" es, en consecuencia, una investigación que no puede inquirir sobre las posibilidades "alternativas" $y$, al no facilitar la comprensión que pueda darle forma al desarrollo de estas, es probable que contribuya principalmente a consolidar los valores encarnados actualmente en las instituciones dominantes ${ }^{17}$. Así, el compromiso con estos valores pone barreras en el camino de la investigación, las cuales 
podrían confirmar que hay posibilidades (las liberadoras, por ejemplo) que no están admitidas dentro de las instituciones dominantes, de tal manera que las demandas el conocimiento que actualmente pueden confirmarse, dados los métodos de la "investigación desinteresada", están subordinadas a esos valores ${ }^{18}$. De lo anterior, se sigue que el desinterés no satisface su pretendida función epistemológica y metodológica (Tema 4). Sin embargo, las demandas válidas de la ciencia no están subordinadas a los valores morales y sociales; su fundamento se encuentra únicamente en las relaciones apropiadas con los datos empíricos y otros factores epistemológicos relevantes ${ }^{19}$. Empero, lograr el acceso a los datos relevantes (esto es, aquellos que están relacionados con las aniomalias) y la imaginación para considerar las posibilidades "alternativas" depende del cultivo de las virtudes morales enunciadas anteriormente.

\section{Conclusión}

Todo lo anterior plantea un gran desafío para la universidad. La universidad, en tanto institución social, necesita de recursos materiales, sociales y financieros: donantes, estudiantes que pagan sus cuotas y disponibilidad de empleos para sus graduados. Las fuentes de esos recursos tienden a estar vinculados estrechamente a las instituciones dominantes de la sociedad, las cuales tienden a valorar a la universidad en la medida en que proporciona el conocimiento y el personal cualificado necesarios para fomentar sus intereses. ¿Podría la universidad diferente de Ellacuría hacerse de los recursos que necesita? Quizá es más prudente preguntarse: ¿Puede institucionalizarse su visión como una parte importante de la universidad contemporánea? (La reflexión sobre la universidad se torna un caso particular de exploración de las posibilidades sociales futuras). Si la respuesta es negativa, la tarea de entender la realidad social que tiene la universidad permanecerá inane y su aporte potencial para avanzar en la justicia y la paz permanecerá sin actualizarse.

NOTAS

1. Ellacuría: "Diez años despućs: écs posible una universidad distinta", en Escritos universitarios, UCA Editores, San Salvador, 1999. Ver los otros ensayos cllacurianos sobre la universidad compilados en Escritos universitarios, "El 
desafío de las mayorías populares" y "Universidad, derechos humanos y mayorias populares", asi como "The task of a Christian university", incluido en el volumen de J. Sobrino et al., Companions of Jesus: The Jesuit Martyrs of El Salvador, Maryknoll: Orbis, 1990.

2. Ver J. Hasset y H. Lacey, "Comprehending reality from the perspective of the poor", prólogo a Towards a Society That Serves Its People: The Intellectual Contribution of El Salvador's Murdered Jesuits [De aquí en adelante, TSSP], Washington: Georgetown University Press, 1991.

3. H. Lacey, "Notes on the Dialectic of Truth and Justice", en B. Schwartz (ed.), Educating for Social Responsibility in a Multicultural World. The Swarthmore Papers 1 (1993), 107-116.

4. "Utopía y profetismo en América Latina. Un ensayo concreto de soteriología histórica", en Escritos teológicos (II). UCA Editores, San Salvador, 2000.

5. Ver H. Lacey, Is Science Value Free? Values and scientific understanding, London: Routledge, 1999: capítulo 5.

6. Ver H. Lacey y B. Schwartz, "Behaviorism, intentionality and socio-historical structure", Behaviorism 14 (1986), 193-210; y "The formation and transformation of values", en W. O'Donohue y R. F. Kitchener (eds.), The philosophy of psychology, London: Sage, 1996. Estos artículos pueden encontrarse también en H. Lacey, Psicologia Experimental e Natureza Humana: Ensaios de Filosofia da Psicologia, Florianópolis (Brasil): Universidade Federal de Santa Catarina, 2001.

7. Ver H. Lacey, "Neutrality in the social sciences: On Bhaskar's argument for an essential emancipatory impulse on social science", Journal for the Theory of Social Behavior (1997), 213-241.

8. Ignacio Martín-Baró, "Hacia una psicología de la liberación”, en Boletín de Psicología Número 22, 219-231, UCA Editores, San Salvador, 1986. En otra parte, he defendido el argumento de que las organizaciones populares se caracterizan por lo siguiente: (1) Sus luchas están arraigadas en identidades grupales; (2) Combinan las formas de organización sindical y las políticas de alianzas; (3) Tienden a fijar sus objetivos en el lenguaje de los distintos derechos humanos; y (4) Luchan por una unidad "orgánica" entre las reivindicaciones concretas de reformas y la búsqueda del cambio estructural. Para mayores detalles, cfr. $\mathrm{H}$ Lacey, "The legacy of El Salvador's Murdered Jesuits", Journal for Peace and Justice Studies (1995), $\mathrm{N}^{\circ} 2,113-126$.

9. Para ver el desarrollo de este razonamiento, ver Lacey (1997), op. cit.

10. H. Lacey, "Alternativas à tecno-ciència e os valores do Forum Social Mundial», en I. Loureiro y J. Correa (eds.), O Espirito do Porto Alegre, São Paulo: Paz è Terra (En prensa).

11. J. Sobrino, Spirituality of Liberation: Toward Political IIoliness, Maryknoll: Orbis, 1988.

12. Ver, además, H. Lacey, "Listening to the evidence: service activity and understanding social phenomena", en C. D. Lisman e I. Harvey (eds.), Beyond the Tower: Concepts and Models for Service Learning in Philosophy, Washinton, D.C.: American Association for Higher Education, 2000.

13. Lacey (1993), op. cit.

14. Las ideas de este párrafo se desarrollan con mayor profundidad en Lacey (2000), op. cit.; ver también Lacey (1993), op, cit. 
15. En aquellas circunstancias donde la universidad contrata empleados (por ejemplo, para los servicios de alimentación, mantenimiento y jardinería) de la comunidad, es especialmente importante que sus salarios, bencficios y condiciones de trabajo se determinen en virtud de principios razonables de justicia. No desarrollaré este punto aquí, pero necesita integrarse con las cuatro condiciones apuntadas.

16. Los escritos de Sobrino sobre el tema de la espiritualidad, que desarrollan en parte ideas de Ellacuría, son particularmente útil (Ver Sobrino, op. cit.)

17. He discutido en detalle en otra parte que las naciones de desinterés (y semejantes) conducen a aproximaciones a la investigación científica (incluyendo las ciencias naturales) que exhiben relaciones mutuamente reforzadoras junto a los valores modernos que busian el control de los objetos naturales. Ver H. Laccy, Is Science Value Free?, op. cit.; "The ways in which the sciences are and are not value free", en P. Gardenfors, K. Kijania-Placek y J. Wolenski (Eds.), actas del $X I$ International Congress of Logic, Metbodology and Philosophy of Science, Dordrecht: Kluwer, 2002.

18. Los detractores (incluyendo aquellos que planearon su asesinato) han acusado a las ideas de Ellacuría implican la politización de la universidad, al punto que esta acusación yace sobre el supuesto que él supeditaba la verdad a los valores políticos, o a los intereses de programas partidarios, es simplemente falsa. Pero sostener que la verdad no puede perseguirse salvo en una interacción dialéctica con algunos valores sociales es algo que tiene muchas consccuencias políticas. Esto provoca el contraargumento de que sus detractores son proclives a subordinar la verdad a los valores que invocan a fin de legitimar sus intereses. Esto se encuentra vinculado con una idea que Ellacuría reiteraba en muchas ocasiones: la injusticia es un obstáculo en la búsqueda de la verdad. Defender la primacía de la dialéctica de verdad y justicia no implica negar que hay también una dialéctica de verdad y valores. Sin duda, reconocer esto es crucial si los resultados de la propia aproximación se someten al escrutinio empírico y teórico más riguroso. No es una verdad a priori el que haya posibilidades liberadoras. La existencia de estas posibilidades necesita demostrarse en la investigación y en la práctica (Uno no puede deducir la verdad de los valores, pero los valores apuntan hacia lo que merece investigarse y hacia los hechos significativos). Relacionado con esto, los dos supuestos metodológicos apuntados arriba (que tienden a ser negados en una buena parte de las corrientes investigativas principales de las ciencias sociales - lacey, 1997, op. cit.) necesitan vindicarse en el transcurso de una investigación exitosa que necesitará compararse con los resultados de otras aproximaciones. La universidad de Ellacuria no niega espacios para otras aproximaciones, pero insiste en la legitimidad e indispensabilidad de la dialéctica de verdad y justicia. En otra parte, en el contexto de la discusión sobre las ciencias físicas y biológicas, he propugnado por la centralidad de aquellas aproximaciones a la investiació que proporcionan un refuerzo mutuo a jas relaciones con los valores de la "participación popular", mientreas señalan la necesidad (basada tanto en consideraciones metodológicas como en los intereses de la democracia) de tener una pluralidad de aproximaciones (lacey, 1999; en prensa, op. cit.)

19. Esto se discute en detalle en los trabajos citados en la nota anterior. 